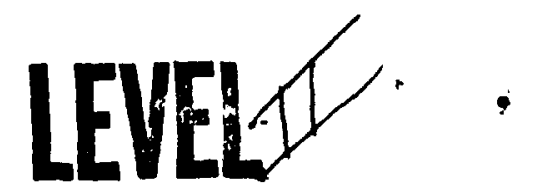

AN ITERATIVE METHOD FOR SOLVING

EIECTROSTATIC PROBIEMS

Tapan K. Sarkar

and

Sadasiva M. Rao

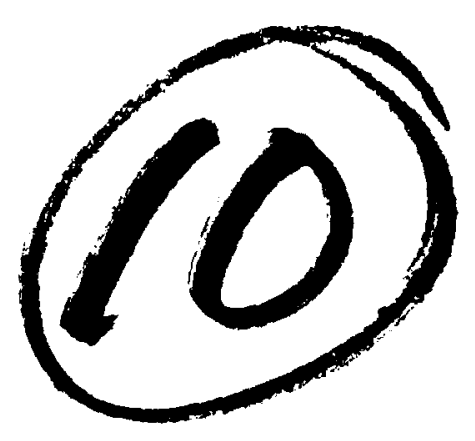

$\nabla$

ins

ivas

$=$

$-$

$x$

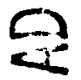

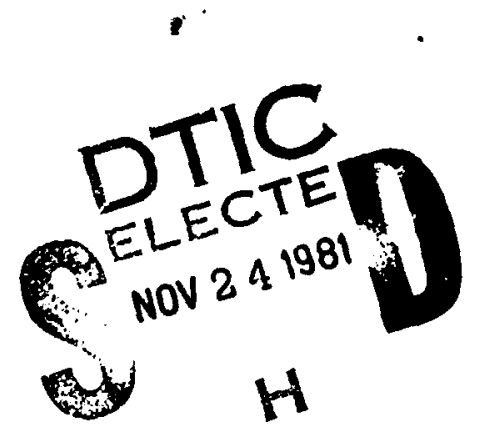

This work has been supported in part by Office of Naval Research under contract N00014-79-C-0598

The authors are with the Electrical Engineering Department Rochester Institute of Technology Rochester, New York 14623
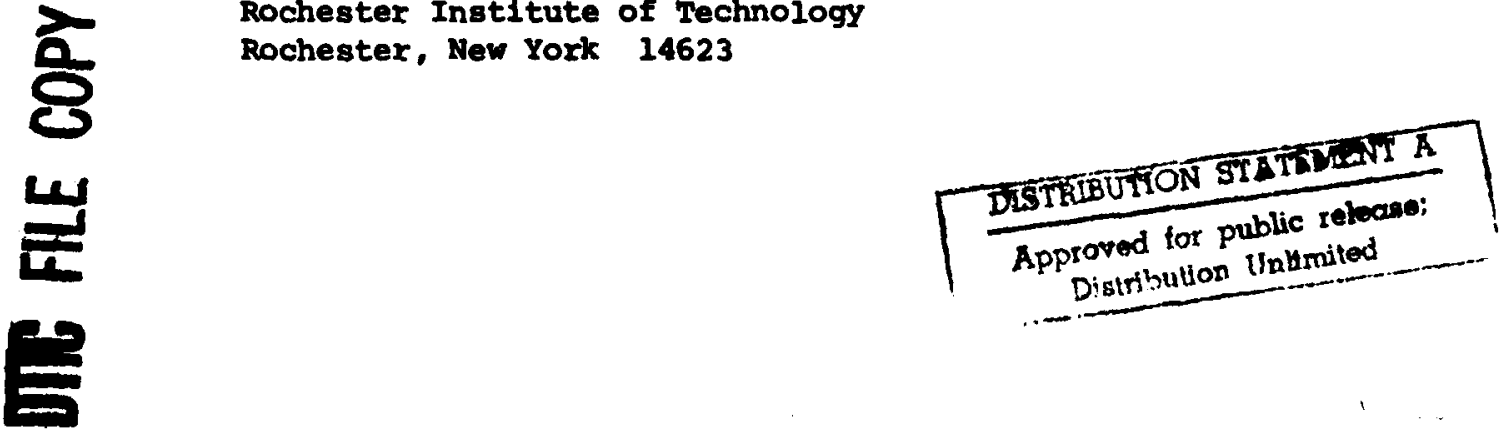
UNCLASSIFIED

SECURITY CLASSIFICATION OF THIS FAGE (rman Dete Entored)

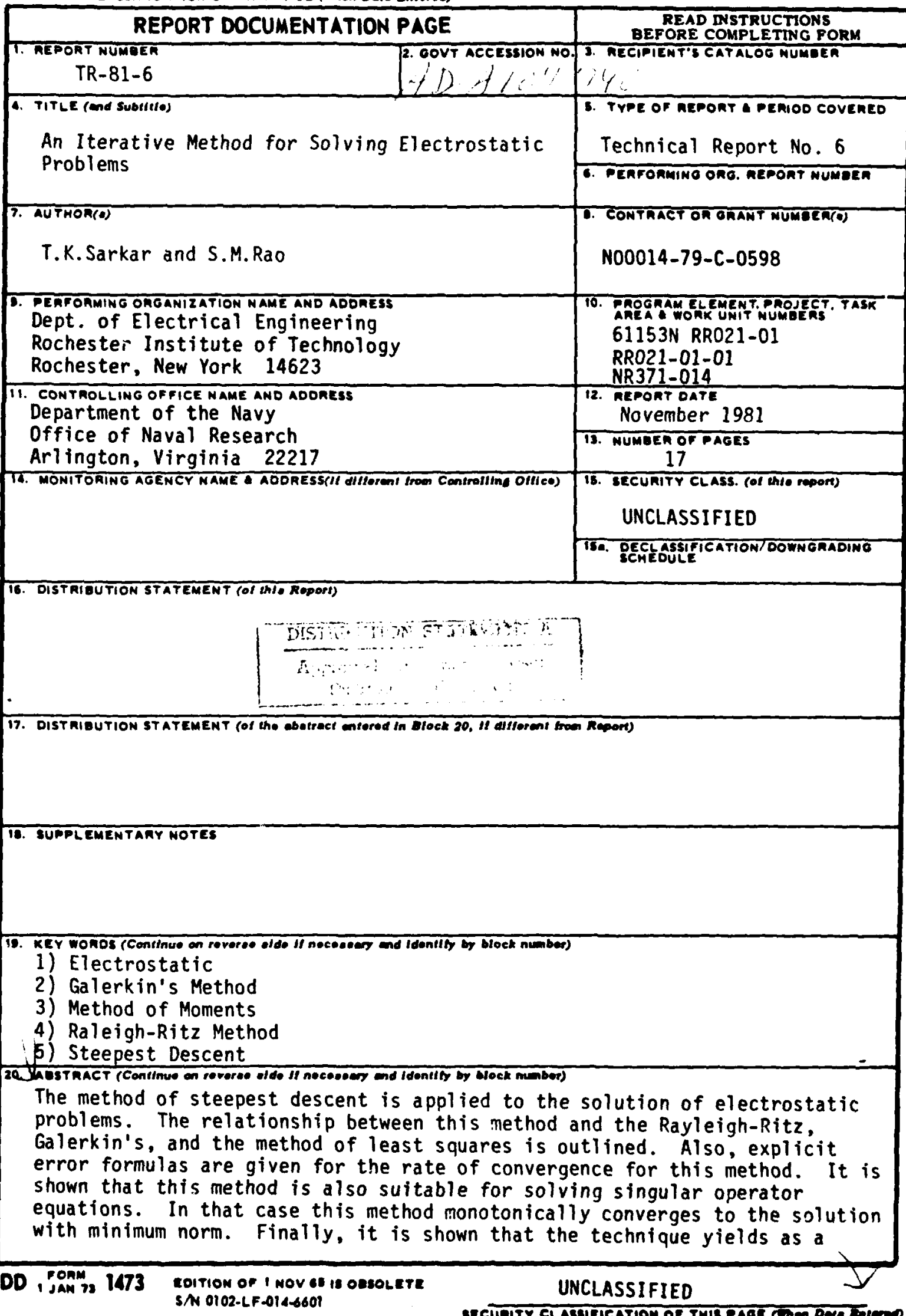


UNCLASSIFIED

SECURitY CLASSIFICATION OF TMIS PAGE (mimer Dele EnIorods

20.

by-product the smallest eigenvalue of the operator in the finite dimensional space in which the problem is solved. Numerical results are presented only for the electrostatic case to illustrate the validity of this procedure which show excellent agreement with other available data,

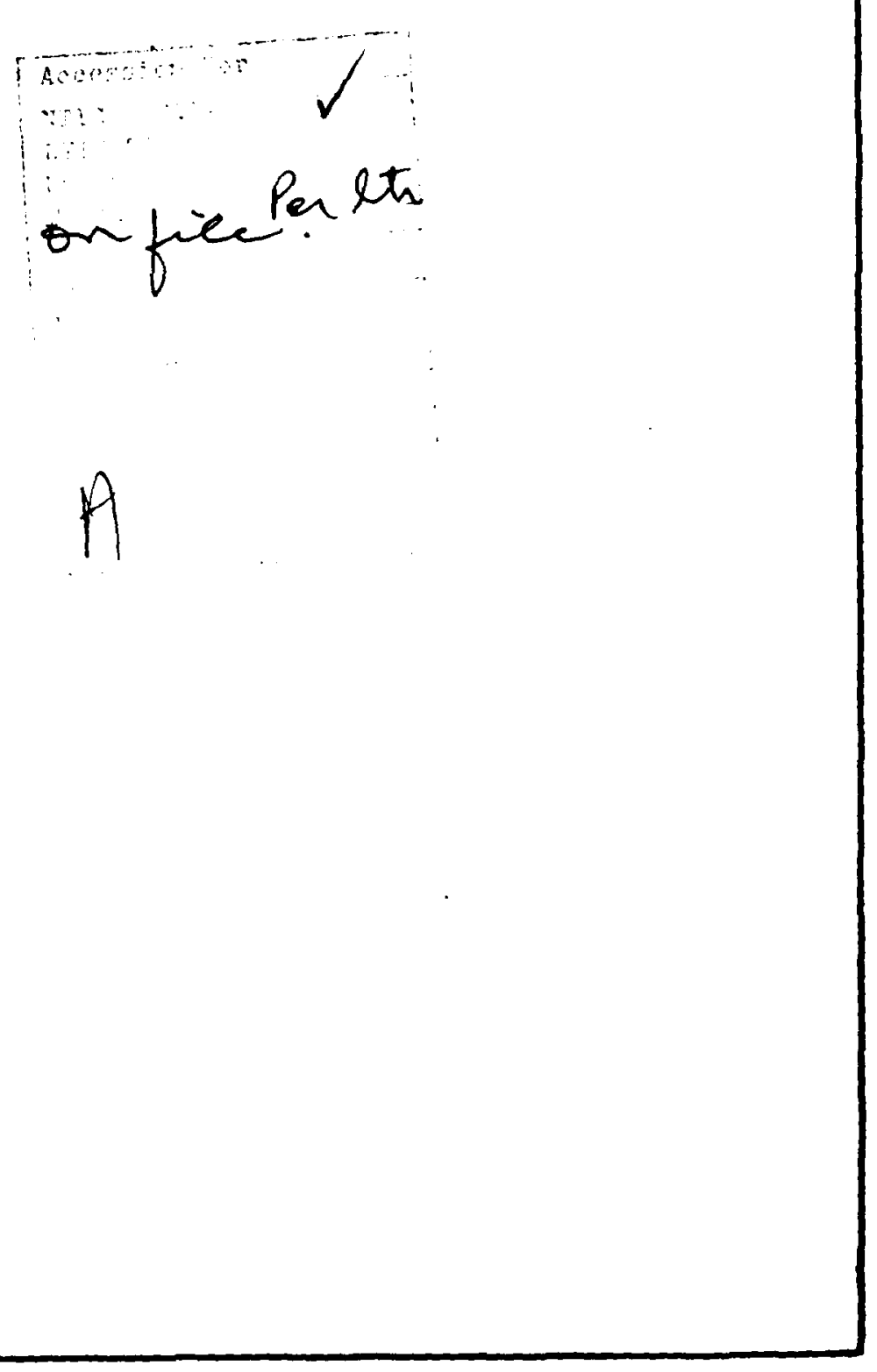

UNCLASSIFIED

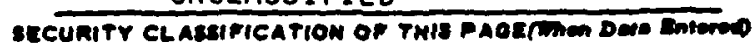


1. INTRODUCTION

Most problems of electromagnetic theory can be formulated in terms of an operator equation

$$
A x=y,
$$

where in general $A$ is an integro-differential operator and $x$ is the unknown to be obtained for the particular excitation $y$.

The approximate methods of solving integro-differential equations of electromagnetics are varied in respect to the ideas lying at their foundation. A number of methods (Variational method, Rayleigh-Ritz method, Galerkin's method, method of moments, method of least squares, and so on) has been elaborated. At the same time the analysis of these methods, particularly in electromagnetic theory, has not advanced far. Most methods have remained without any theoretical analysis and have only been verified by their effectiveness in individual examples.

As is generally known, the majority of the linear problems of analysis may be reduced to the problem of the extremem of quadratic functionals. This fact may be utilized on the one hand for different theoretical investigations relating to these problems (theorems of the existence of a solution, properties of the eigenvalues and so on). On the other hand it serves as a basis for direct methods for solving the problems named.

In this paper a certain method of successive approximations - the method of steepest descent - for the solution of problems concerning the minimum for quadratic functionals and of the linear problems connected with them is elaborated. 
The idea of this method goes back to Cauchy, who proposed it in 1895 for the solution of the problems of the minimum of a function of $\mathrm{N}$ variables, and a problem similar to that: the solution of a system of $\mathrm{N}$ algebraic equations in $\mathrm{N}$ unknowns. Other attempts at developing the ideas of Cauchy have been made in the works of Temple [1], Courant [2] and Kantorovich [3] who provided the mathematical foundation of the method of steepest descent.

The principle distinction between the method of steepest descent and the method of Rayleigh-Ritz, Galerkin's, and the method of least squares is that the sequence of approximations is not obtained in a form selected a priori, but in a form determined by the problem itself. From the Neumann series or the Picard's method of successive approximations [4] this method is distinguished by the size of the region of convergence - which in a number of important cases coincides with the region of existence of the solution - as well as by the nonlinear character of the dependence of successive approximations.

During the theoretical investigation of every approximate method, the following three problems generally arise, in the order of increasing accuracy and difficulty:

(a) The establishment of convergence.

(b) The investigation of the rapidity of convergence.

(c) An effective estimate of error.

The solution of these problems is quite complex and demands a special individual approach in each instance.

In this paper we present the mathematical foundation of the method of steepest descent and apply it to the solution of electrostatic field problems. It is noted that this method requires considerably less computer storage than the method of moment formulation using 
subdomain expansion and testing [11]. Since the method is iterative a good guess may considerably reduce the computation time.

Since electrostatic problems represent the static limit (frequency approaches zero; of the corresponding electromagnetic problems, the problems treated in this paper therefore represent the first step towards the development of this iterative technique for the electrodynamic case.

2. THE METHOD OF STEEPEST DESCENT

We start the description of this method by defining a quadratic functional $I(x)$ in a linear normed space. The functional $I(x)$ is defined by

$$
I(x)=\langle r, S r\rangle,
$$

where the inner product is defined as

$$
\langle C, D\rangle=\int_{x} C(x) \bar{D}(x) d x \text {. }
$$

In (3) $\bar{D}(x)$ denotes the complex conjugate of $D(x)$ and the norm of $C$ is given by

$$
\|c\|^{2}=\langle c, c\rangle=\int i c^{2}(x) \mid d x \text {. }
$$

In (2) $S$ is a hermitian positive definite operator and

$$
r=A x-y
$$

Sy a quadratic we mean a functional $I(x)$ such that the expression $I(x+\alpha p)$ is a polynomial of second degree in $\alpha$, whatever elements $x$ and $p$ may be. In seeking the minimun of the quadratic functional I (x+ap) we use an arbitrary element $x_{0}$ as an initial approximation and the direction of the gradient at the point $x_{0}$ is given by

$$
\frac{d}{d \alpha}\left[I\left(x_{0}+\alpha p\right)\right]=\frac{d}{d \alpha}[\langle r, S r>+<\alpha S A p, r>+<S r, \alpha A p>+<\alpha S A p, \alpha A p>] \text {. }
$$

We select a normed element $p=p_{0}$, so that the derivative in (6) for $\alpha=0$ will be a maximum. Since $I\left(x_{0}+\alpha p\right)$ is a polynomiai of second degree in $\alpha$, for a certain $\alpha=\alpha_{0}$, it will attain a minimum value. The element $x_{1}=x_{0}-\alpha_{0} p_{0}$ we adopt as the next approximation, in iurn, after which the 
iteration becomes

$$
x_{n+1}=x_{n}-\alpha_{x} p_{n}
$$

So from the starting point $x_{n}$, the approximation proceeds in the direction $p_{n}$ for a distance $\alpha_{n}$ along the surface $I\left(x_{n}\right)$, attempting to choose $\alpha_{n}$ and $p_{n}$ at each step so as to approach the minimum point of $I(x)$. The best choice of $p_{n}$ would of course be in the direction of the error vector $x_{e}-x$, but this vector is unfortunately not krown. Here $x_{e}$ is the exact solution. In the next section it is shown how to select $P_{n}$ for the various functionals $I(x)$ that are associated with the method of steepest descent.

Next we compute the optimum step length $\alpha$ in the direction of $p$ such that the quadratic functional $I(x+\alpha p)$ is minimized. This implies that in (6)

$$
\frac{d}{d \alpha}\left[I\left(x+\alpha_{p}\right)\right]=0
$$

resulting in

$$
\alpha=\frac{\langle r, S A p\rangle}{\langle A p, S A p\rangle}
$$

In the next section, for various search directions $p$, the optimum values of $\alpha$ are given.

The process under discussion may be interpreted geometrically. First let the space in which the functional $I(x)$ is defined be two dimensional. The contour lines $I(x)$ are, in general, a family of similar ellipses with center at the minimum point. The point $x_{0}$, with which we began the process, lies in a certain ellipse of the family, viz. on $I(x)=I\left(x_{0}\right)$. From It we go in the direction of the gradient, I.e. along the normal to this ellipse, to a point corresponding to the least value $I(x)$ on this normal, i.e. to the point $x_{1}$, where it touches (is tangent to) some ellipse of the family. From this point we go along the normal to the ellipse $I(x)=I\left(x_{1}\right)$ and so forth. If the number of dimensions of the space be more than two, in plac 
a family of ellipses there is a family of ellipsoids, and instead of the contour there are surfaces. However, each step of the process may be examined in a plane, viz., the plane that passes through the minimum point and the points $x_{0}$ and $x_{1}$. The sections of the ellipsoids by the plane will be families of ellipses resembling the two dimensional case.

\section{DEVELOPMENT OF THE METHOD OF STEEPEST DESCENT}

Several different functionals are available as error measures to be used in conjunction with the method of steepest descent. The three most popular functionals are described along with the corresponding versions of the method of steepest descent. We also note the limitations associated with each of the functionals. In the development of this section we assume $A x=0$ in (1) has only the zero solution. This restriction is relaxed in the next section, however.

(a) Minimization in the domain of $A$ In this case, the functional is chosen as

$$
\begin{aligned}
J_{a}(a) & =\left\langle x-x_{e}, x-x_{e}\right\rangle \\
& =\left\langle A^{-1}(A x-y), A^{-1}(A x-y)\right\rangle=\left\langle A^{-1} r, A^{-1} r\right\rangle \\
& =\left\langle r,\left[A^{-1}\right] *\left[A^{-1}\right] r\right\rangle,
\end{aligned}
$$

where

$$
\begin{aligned}
x_{e} & =\text { exact solution } \\
r & =A x-y \\
S_{a} & =\left[A^{-1}\right] *\left[A^{-1}\right] \\
& {[A] *=\operatorname{adjoint} \text { of }[A] }
\end{aligned}
$$

For this functional, at each iteration the error between the approximate solution $x_{n}$ at the end of $n$ iterations and the exact solution $x_{e}$ is made smaller than the error between $x_{n-1}-x_{e}$. The convergence is monotonic as proved in the next section. However, the residuals may 
oscillate. This implies that there is no guarantee whatsoever that

$$
\left\|A x_{n}-y\right\|=\left\|r_{n}\right\|>\left\|r_{n+1}\right\|=\left\|A x_{n+1}-y\right\| \text { for all } n \text {. }
$$

The method of steepest descent for the functional $I_{a}(x)$ is described below. The directions $p_{n}$ are chosen as

$$
P_{n}=A^{*} r_{n} \text {. }
$$

so from (7) and (13), $\alpha_{n}$ is given by

$$
\begin{aligned}
\alpha_{n} & =\frac{\left\langle r_{n}, S_{a} A p_{n}\right\rangle}{\left\langle A p_{n}, S_{a} A p_{n}\right\rangle} \\
& =\frac{\left\langle r_{n}, r_{n}\right\rangle}{\left\langle A * r_{n}, A * r_{n}\right\rangle} \quad \text { [utilizing(1)] } \\
& =\frac{\left\|r_{n}\right\| \|^{2}}{\| A * r} \|_{n}^{2}
\end{aligned}
$$

and the iteration is given by

$$
\begin{aligned}
x_{n+1} & =x_{n}-\frac{\left\|r_{n}\right\|^{2}}{\left\|A * r_{n}\right\|^{2}} A * r_{n} \\
& =x_{n}-\frac{\left\|A x_{n}-y\right\|^{2}}{\left\|A *\left[A x_{n}-y\right]\right\|^{2}} A *\left[A x_{n}-y\right] .
\end{aligned}
$$

It is important that if $y \& R(A)$ the iteration defined by (15) does not converge at all. Here $R(A)$ is defined as the range space of $A$.

(b) Minimization in the range of $A$

For this case

$$
I_{b}(x)=\langle r, r\rangle=\langle A x-y, A x-y\rangle
$$

which implies

$$
S_{b}=\text { Identity operator. }
$$

However, there are two versions of this particular method of steepest descent depending on the choice of $P_{n}$. The choice of $P_{n}$ for this case depends also on the operator $A$. 
(i) If $A$ is positive/negative definite, then we choose $p_{n}=r_{n} \cdot$

From (7) and (18) $\alpha_{n}$ is given by

$$
a_{n}=\frac{\left\langle r_{n}, A r_{n}\right\rangle}{\left\langle A r_{n}, A r_{n}\right\rangle}
$$

and the iteration for this special operator is defined by

$$
\begin{aligned}
x_{n+1} & =x_{n}-\frac{\left\langle r_{n}, A r_{n}\right\rangle}{\left\langle A r_{n}, A r_{n}\right\rangle} r_{n} \\
& =x_{n}-\frac{\left\langle A x_{n}-y, A\left[A x_{n}-y\right]\right\rangle}{\left\|A\left(A x_{n}-y\right)\right\|^{2}}\left[A x_{n}-y\right] .
\end{aligned}
$$

(ii) If $A$ is an arbitrary operator, we choose

$P_{n}=A * r_{n}$ [same as in (13)].

For this particular choice of $p_{n}$, and utilizing (17) we get

$$
\alpha_{n}=\frac{\left\langle r_{n}, A A * r_{n}\right\rangle}{\left\langle A A * r_{n}, A A * r_{n}\right\rangle}=\frac{\left\|A * r_{n}\right\|^{2}}{\left\|A A * r_{n}\right\|^{2}} .
$$

The method of steepest descent is then described by the iteration

$$
\begin{aligned}
x_{n+1} & =x_{n}-\frac{\left.\left\|A * r_{n}\right\|\right|^{2}}{\left\|A A * r_{n}\right\|^{2}} A \div r_{n} \\
& =x_{n}-\frac{\left\|A *\left[A x_{n}-y\right]\right\|^{2}}{\left\|A A \div\left[A x_{n}-y\right]\right\|^{2}} A *\left[A x_{n}-y\right] .
\end{aligned}
$$

It is important to roint out that for both (i) and (ii) the residuals decrease monotonically at each iteration. However, there is no guarantee for an arbitrary operator $A$ that the corresponding errors in the solutions $x_{n}$ would be small. In other words the following inequality is not satisfied:

$$
\left\|x_{n}-x_{e}\right\|>\left\|x_{n+1}-x_{e}\right\| \text { for all } n \text {. }
$$


The method of steepest descent described by $(20)$ or $(22)$ converges to the exact solution if $y \in R(A)$. If $y \notin R(A)$ then the iterations described by (20) or (22) converge to a least squares solution with the minimum norm. It is interesting to point out that the same functional as described in (16) is also minimized in the application of the method of least squares.

(c) Minimization both in domain and range of $A$.

In the method of steepest descent this choice of the functional works only if $A$ is positive/negative definite. The functional is defined as

$$
\begin{aligned}
I_{c}(x) & =\left\langle x-x_{e}, A\left(x-x_{e}\right)\right\rangle \\
& =\left\langle A^{-1} r, r\right\rangle=\left\langle r,\left[A^{-1}\right] \div r\right\rangle
\end{aligned}
$$

and therefore

$$
s_{c}=\left[A^{-1}\right] \% \text {. }
$$

In this case we select the paths $P_{n}$ as

$$
p_{n}=r_{n} \text { [same as in (18)]. }
$$

Utilizing (7) and (18) we obtain

$$
\alpha_{n}=\frac{\left\langle r_{n}, r_{n}\right\rangle}{\left\langle r_{n}, A r_{n}\right\rangle}=\frac{\left\|r_{n}\right\|^{2}}{\left\langle r_{n}, A r_{n}\right\rangle} .
$$

The iterative method is then defined by

$$
\begin{aligned}
x_{n+1} & =x_{n}-\frac{\left\|r_{n}\right\|^{2}}{\left\langle r_{n}, A r_{n}{ }^{2}\right.} r_{n} \\
& =x_{n}-\frac{\left\|A x_{n}-y\right\|^{2}}{\left\langle A x_{n}-y, A\left[A x_{n}-y\right]\right\rangle}\left[A x_{n}-y\right] .
\end{aligned}
$$

The iterations defined by (27) converge to the exact solution if and only if
(1) A is positive/negative definite, and
(2) $y \in R(A)$ 
If either of these two conditions is violated, the convergence of (27) is not assured.

It is interesting to note that the same functional $I_{c}(x)$ defined by (24) is minimized in the application of the variational - Rayleigh-Ritz and Galerkin's methods. From the above discussion it thus becomes clear that in Rayleigh-Ritz and Galerkin's methods there is no guarantee of the convergence of the solutions as $n \rightarrow \infty$ if either of the conditions in (28) is violated. An example has been presented in the Appendix, to illustrate the above phenomenon, when none of the conditions in (28) are satisfied.

4. RATE OF CONVERGENCE OF THE METHOD OF STEEPEST DESCENT

The method of steepest descent utilizes the iterative process $x_{n+1}=x_{n}-\alpha_{n} p_{n}$.

In order to stop the iterative process, we make sure that the residuals are below a certain small value, i.e.

$$
\left\|r_{n}\right\|=\left\|A x_{n}-y\right\|<\varepsilon .
$$

In practice, we have found specifying this criterion is equivalent to restricting

$$
\left\|\alpha_{n}-\alpha_{n+1}\right\|<\varepsilon \text {. }
$$

$i . e$, the values of $\alpha_{n}$ have settled to a stable numerical value. observe that for the iterative processes described by $(20),(22)$, and (27) the value of $\alpha_{n}$ has settled to the sriallest eigenvalue of the operator $A$ in the finite dimensional space in which the problem is being solved. For the iterative process in (15) $L_{n \rightarrow \infty} \alpha_{n}$ yields the smallest eigenvalue of the adjoint operator $A^{*}$ in the finite dimensional space in which the problem is represented.

The next question that automatically rises is how fast do these $\alpha_{n}$ converge, or $x_{n}$ 's approach the true solution $x_{e}$, or the residuals $r_{n}$ converge to zero. These questions are answered by the following theorems. 
Theorem 1: The sequence $\left\{x_{n} \ln _{n}=0,1,2, \ldots\right\}$ generated by (15) for the functional $1_{a}(x)$, derived by the method of steepest descent satisfies

$$
\left\|x_{n}-x_{e}\right\| \leq\left\{\frac{\left\{\|A\| \cdot\left\|A^{-1}\right\|^{2}\right\}^{-1}}{\left.\|\| A\|\cdot\| A^{-1} \|\right\}^{2}+1}\right\}^{n} \cdot\left\|x_{0}-x_{e}\right\| .
$$

The proof of this theorem can be found in [5-7]. The proof assumes that $y \in R(A)$, otherwise (15) does not converge.

Theorem 2: The sequence $\left\{x_{n}\right\}$ generated by (22) for the functional $I_{b}(x)$, derived by the method of steepest descent satisfies

$$
\left\|x_{n}-x_{e}\right\| \leq\left\|A^{-1}\right\|\left\{\frac{\left\{\|A\| \cdot\left\|A^{-1}\right\|^{2}-1\right.}{\left\{\|A\| \cdot\left\|A^{-1}\right\|\right\}^{2}+1}\right\}^{n} \cdot\left\|A * A x_{0}-A * y\right\| \cdot
$$

The proof of this theorem is given in [5-7].

The estimate $(32)$ is valid even if $y \in R(A)$. In that case $A^{-1}$ should be interpreted as the generalized inverse of $A$.

Theorem 3: The sequence $\left\{x_{n}\right\}$ generated by (27) for the functional $I_{c}(x)$, derived by the method of steepest descent satisfies

$$
\left\|x_{n}-x_{e}\right\| \leq\left\{\frac{\|A\| \cdot\left\|A^{-1}\right\|-1}{\|A\| \cdot\left\|A^{-1}\right\|+1}\right\}^{n} \cdot\left\|x_{0}-x_{e}\right\| .
$$

The proof of this theorem can be obtained from [8].

Observe that out of the three estimates (31), (32) and (33), the last one has the best rate of convergence. However, it is only applicable to a certain restricted class of definite operators $A$.

(31) yields a better rate of convergence than (32). However, the assumption is $y \in R(A)$ - which may be valid for a wide class of problems of interest. However, if $y \notin R(A),(31)$ no longer holds and the only iterative technique that is available is (22). The rate of convergence of $\left\{x_{n}\right\}$ for the iterative method in (22) is given by (32). 
Now if $\left\|A^{-1}\right\|$ is unbounded or if $A^{-1}$ does not exist (when $A x=0$ has a nontrivial solution) then the above three theorems cease to be true entirely, just because the solution cannot exist or is not unique. Under these circumstances, a weaker assertion holds and is given by theorem 4.

Theorem 4: If $A x=0$ has a nontrivial solution, or if $\left\|A^{-1}\right\|$ is unbounded, then each successive approximation is nearer to $x_{e}$ than is the preceeding, i.e.

$$
\left\|x_{n+1}{ }^{-x_{e}}\right\|^{2} \leq \frac{\|A\|^{2} \cdot c \cdot\left\|x_{0}-x_{e}\right\|^{2}}{\|A\|^{2} \cdot c+n\left\|x_{0}-x_{e}\right\|^{2}},
$$

where $C$ are certain constants which depend on $x_{0}$ and $y$, and

$$
r_{n}-r_{n+1} \geq Q r_{n}^{2} \text {, where } Q \text { is a constant. }
$$

The proof of (34) is given in [9] and that of (35) in [3]. From (35) it is seen that the numbers $r_{n}$ diminish, have a finite limit and by passing to the limit in (27) one is easily convinced that the limit is equal to zero. On this basis of inequality (35) it is seen that [3]

$$
r_{n}=\theta(1 / n)=\text { [of the order of } 1 / n \text { ] }
$$

and hence the rate of convergence is of the order of $1 / n$, which is explicitly given by (34).

5. NUMERICAL RESULTS

In this section, the method of steepest descent, described so far, is applied to the solution of electrostatic problems. In this case the operator $A$ is real and self adjoint. Hence we have used the iterative method given by (27). The geometries considered here are a thin straight wire and a $90^{\circ}$ bent wire raised to a specified potential. The numerical results are compared with the method of moment solution [10] and excellent 
agreement is obtained in each case. A considerable reduction in the computer storage is also noted with the present method which makes it especially attractive for large electromagnetic field problems. Since the method is iterative, a good initial guess may significantly reduce the computation time.

\section{Straight Wire}

In Table I we present the charge induced on a thin straight wire of length $1.0 \mathrm{~m}$ and radius $0.001 \mathrm{~m}$ raised to a constant potential $1 / 4 \pi \varepsilon_{0}$ volts, where $\varepsilon_{0}$ is the free space permittivity. The wire is divided into nine equal subsections and charge is computed at the center of the subsection. As is evident from the table, both solutions show excellent agreement up to at least four decimal places.

The present solution with the method of steepest descent is obtained by assuming a constant charge distribution as initial guess and the method converged in 16 iterations. The smallest eigenvalue of the integral operator was obtained as .10285 as a by-product.

We have also solved the problem of charge distribution on a $3000 \mathrm{~m}$ long wire with $001 m$ radius charged to $1 / 4 \pi \varepsilon_{0}$ volts. The method converged in 29 iterations.

Bent Wire

In Table 2, we present the charge induced on a $90^{\circ}$ bent wire raised to a potential $1 / 4 \pi \varepsilon_{0}$ volts. Each section of the wire is $1.0 m$ long and the radius is $0.001 \mathrm{~m}$. The wire is divided into 20 unequal intervals with more subsections in the vicinity of the bend. For comparison, we present the solution obtained by the method of moments using pulse expansion and point matching. In Table 2 , the parameter $\ell$ is measured along the length of the wire with the bend at $l=0$. Again both the 
TABLE I

Charge induced (coulombs $/ \mathrm{m}$ )

$\begin{array}{lll}\ell \text { (meters) } & \text { Method of Moments } & \begin{array}{l}\text { Method of Steepest } \\ \text { Descent }\end{array} \\ -0.444 & 0.94083 \mathrm{E}-01 & 0.94084 \mathrm{E}-01 \\ -0.333 & 0.83065 \mathrm{E}-01 & 0.83065 \mathrm{E}-01 \\ -0.222 & 0.80153 \mathrm{E}-01 & 0.80154 \mathrm{E}-01 \\ -0.111 & 0.78897 \mathrm{E}-01 & 0.78897 \mathrm{E}-01 \\ 0.0 & 0.78532 \mathrm{E}-01 & 0.78532 \mathrm{E}-01 \\ 0.111 & 0.78897 \mathrm{E}-01 & 0.78897 \mathrm{E}-01 \\ 0.222 & 0.80153 \mathrm{E}-01 & 0.80154 \mathrm{E}-01 \\ 0.333 & 0.83065 \mathrm{E}-01 & 0.83065 \mathrm{E}-01 \\ 0.444 & 0.94084 \mathrm{E}-01 & 0.94084 \mathrm{E}-01\end{array}$

TABLE 2

Charge Induced (Coulombs/m)

$\ell$ (meters)

Method of Moments

Method of Steepest Descent

$-0.930$

0.89847267 E-01

0.89848161 E-01

$-0.790$

$0.78931868 E-01$

0.78932703 E-01

$-0.650$

0.75660667 E-01

$0.75661421 E-01$

$-0.510$

$0.73936105 E-01$

0.73936880 E-01

$-0.370$

$0.73649168 E-01$

$0.73649943 E-01$

$-0.270$

$0.61391938 E-01$

0.61392322 E-01

$-0.210$

$0.62429000 E-01$

$0.62429097 E-01$

$-0.150$

$0.62455501 E-01$

$0.62456008 E-01$

$-0.090$

0.62318593 E-01

0.62318966 E-01

$-0.030$

$0.62107146 E-01$

0.62107623 E-O1

0.030

0.62107511 E-01

0.62107649 E-01

0.090

$0.62318355 E-01$

0.62318619 E-01

0.150

0.62455675 E-OI

0.62455688 E-01

0.210

0.62428676 E-OI

0.62428772 E-01

0.270

0.61391711 E-01

0.61391935 E-O1

0.370

0.73648870 E-OI

0.73649585 E-01

0.570

$0.73940277 \mathrm{E}-01$

0.73940575 E-01

0.650

0.75661004 E-01

0.75661361 E-01

0.790

$0.78932526 E-01$

$0.78932822 E-01$

0.930

0.89945679 E-01

0.89846134 E-01 
solution procedures show remarkable agreement. Further note the absence of any anomalies in the solution of the method of steepest descent which indicates the capability of the solution procedure to handle abrupt bends.

Again as a by-product we have obtained the smallest eigenvalue of the operator, which is $\mathbf{. 0 9 9 4 3 5}$. The smallest eigenvalue of the operator is important as it may tell us how ill-conditioned the actual problem is. If the smallest eigenvalue turns out to be extremely small then we know that the homogeneous equation might have a nontrivial solution and the corresponding method-of-moments formulation may yield inaccurate results.

Presently work is being carried out on the extension of the method of steepest descent to electromagnetic problems.

\section{CONCLUSION}

The method of steepest descent has been applied to solve electrostatic problems. Explicit error formulas and the rate of convergence of the method of steepest descent is given. It is shown that this method is also sultable for analyzing singular operator equations which may arise in certain electromagnetic problems. In this case the rate of convergence is $(1 / n)$. The method yields as a by-product the smallest eigenvalue of the operator in the finite dimensional space in which the problem is solved. Numerical results for electrostatic problems show excellent agreement between the method of moments formulation and the method of steepest descent. It is also seen that this method requires considerably less storage than the corresponding method of moments formulation with pulse functions as expansion and impulse functions as weighting. Since the method of steepest descent is an iterative method, good initial guess may considerably reduce the computation time. 


\section{REFERENCES}

1. G. Temple, "The General Theory of Relaxation Methods Applied to Linear Systems", Proc. Royal Soclety, London (Series A), Vol. 169, 1939, Pp. 476-500.

2. R. Courant, "Varlational Methods for the Solution of Problems of Equilibrium and Vibrations", Bull. Amer. Math. Soc., Vol. 49, 1943, pp. I-23.

3. L. V. Kantorovich, "Functional Analysis and Applied Mathematlcs", Uspekhi Mat. Nauk, Vol. 6, 1948, no. 3, pp. 89-185.

4. E. Plcard, "Sur un Théoreme General Relatif Aux Equations Intégrals de Premiere Espece et sur Quelques Problémes de Physique Mathematique", Rend. Cir. Mat. Palermo, Vol. 29, 1910, Pp. 79-97.

5. W. J. Kammerer and M. Z. Nashed, "Steepest Descent for Singular Linear Operators with Nonclosed Range", Applicable Analysis, 1971, Vol. 1. PP. 143-159.

6. M. 2. Nashed, "Steepest Descent for Singular Linear Operator Equations", SIAM J. Numer. Anal., Vol. 7, 1970, Pp. 358-362.

7. V.M. Friedman, "On the Convergence of Methods of Steepest Descent Type", Uspekhi Mat. Nauk, Vol. 17, 1962, Pp. 201-204.

8. T. K. Sarkar, K. Siarkiewicz, and R. Stratton, "A Survey of Numerical Methods for Solution of Large Systems of Linear Equations for Electromagnetic Field Problems". (to be published in IEEE-AP)

9. S. F. McCormick and G. H. Rodrigue, "A Uniform Approach to Gradient Methods for Linear Operator Equations", J. of Math. Anal. and Appl., Vol. 49, 1975, Pp. 275-285.

10. R. F. Harrington, "Field Computation by Moment Methods", The MacMillan Company, New York, 1968.

11. S.M. Rao, A.W. Glisson, D.W. Wilicon and B.S. Vidula, "A Simple Numerical Solution Procedure for Stat les Problems Involving Arbitrary-Shaped Surfaces," IEEE Trans. on Ant. and Prop., Vol. AP-27, No. 5, Sept 1979, PP 604-609.

\section{APPENDIX}

In this appendix we illustrate some of the problems associated with the application of a variational-Rayleigh-Ritz and Galerkin"s methods without proper examination of the operator itself. 
As an example consider the solution of the equation $\frac{d x}{d z}=1$ in the region $0 \leq z \leq 1$ with $x(z=0)=0$ The exact solution to this problem is $x$ exact $=z$. Assume that an approximate solution for $z$ is sought as

$$
x=\sum_{n=1,3,5,7, \ldots} a_{n} \sin \frac{n \pi z}{2}
$$

The functions $\sin \frac{n \pi z}{2}$ are orthogonal in the range $0 \leq z \leq 1$ and form a complete set. We can obtain an approximate solution of the above equation using the concepts of Fourier series. In this case we minimize the functional

$$
I(z)=\int_{0}^{1}\left[\frac{d}{d z}\left\{\sum_{\text {odd }} a_{n} \sin \frac{n \pi z}{2}\right\}^{2}-1\right]^{2} d z
$$

The solution is obtained as

$$
x_{F}=\sum_{\text {odd }} \frac{8}{n^{2} \pi^{2}} \sin \frac{n \pi}{2} \sin \frac{n \pi z}{2}
$$

We could have also solved the above differential equation by biind application of Galerkin's method. In that case, we solve the following equations [10]

$$
<\frac{d}{d z} \sum_{\text {odd }} a_{n} \sin \frac{n \pi z}{2}-1 ; \sin \frac{m \pi z}{2}>=0 \text { for } m_{\text {odd }}
$$

The various orders of approximation obtained by Galerkin's method are presented in Figures 1 and 2. Observe that the convergence obtained using Galerkin's method in this case is highly irregular. Observe neither

$$
\operatorname{Lt}_{n \rightarrow \infty}\left\|x_{n}\right\|^{2} \triangleq\left(\int_{0}^{1} x_{n}^{2} d z\right)^{2} \rightarrow\left\|x_{\text {exact }}\right\|^{2} \text { nor } \underset{n \rightarrow \infty}{\operatorname{Lt}}\left\|A x_{n}\right\|^{2}-\|y\|^{2}
$$

monotonically. Yet an approximate solution may be obtained as the dimension of the problem is increased. Note that the error in a one term Fourier series solution is as large as that of a one hundred term Galerkin's method solution.

It is important to point out that a Galerkin's method is not applicable for this problem since the same expansion functions do not form a complete set, both in the domain $\{D(A)\}$ and in the range $\{R(A)\}$ of the operator A. Yet a numerically meaningful result may sometimes be obtained if the order of the approximation is chosen as a large number. In summary, if one formally applies Galerkin's method to a problem for which it is not applicable, one may obtain an approximate solution if a large number of expansion function is chosen. However convergence of the solutions in some cases may be erratic as shown in the figures. 


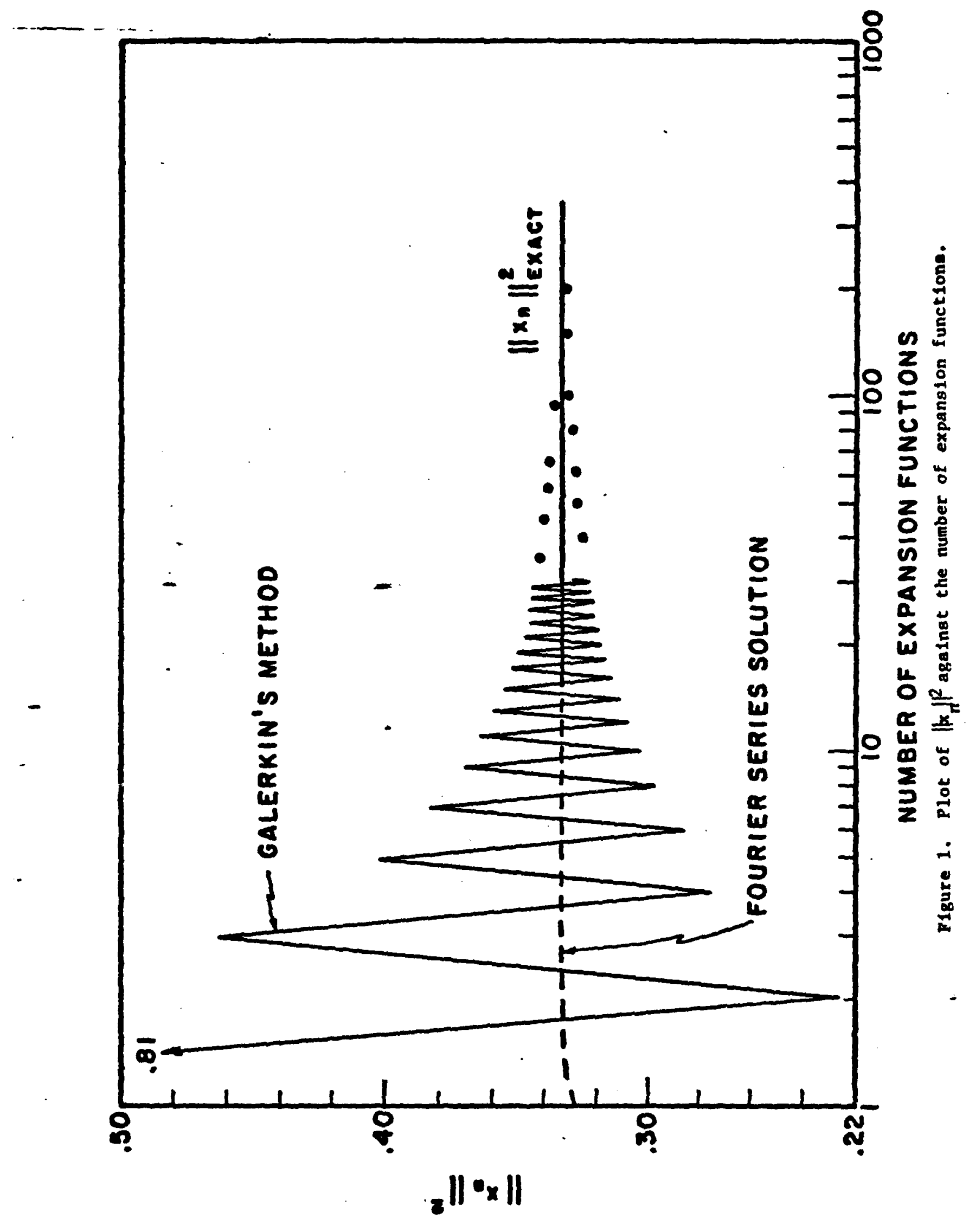




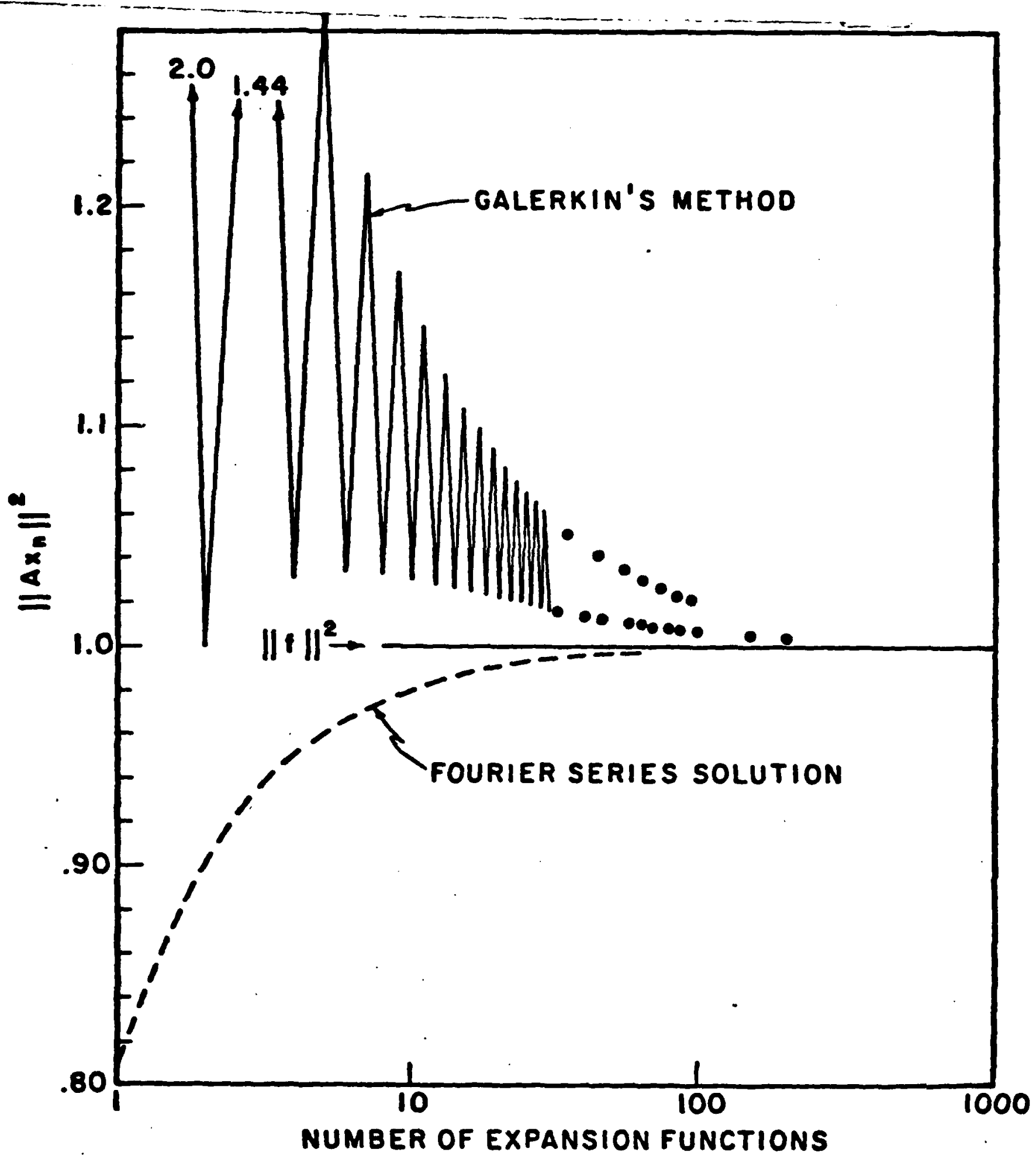

F1gure 2. Plot of $\left\|A x_{n}\right\|^{2}$ for Galerkin's method and the Fourler series oolution against the number of expansion functions. 